\title{
Highly Sensitive Biosensors Based on High-Performance Carbon Nanotube Field-Effect Transistors
}

\author{
Yasuki Yamamoto*, Kenzo Maehashi, Yasuhide Ohno and Kazuhiko Matsumoto \\ The Institute of Scientific and Industrial Research, Osaka University, \\ Mihogaoka 8-1, Ibaraki, Osaka 567-0047, Japan
}

(Received Feburary 28, 2009; accepted June 8, 2009)

Key words: carbon nanotube, field-effect transistor, $\mathrm{pH}$ sensor, biosensor, DNA hybridization, label-free, aptamer, local electrolyte gate, noise, signal-to-noise ratio

Electronic detection of biomolecules has been attracting much interest in the fields of clinical diagnosis, pharmacy and biotechnology. In particular, developing highly sensitive, label-free, cost-effective, simple and disposable sensors is strongly required for home medical care. Carbon nanotube field-effect transistors (CNTFETs) with singlewall carbon nanotube (SWNT) conducting channels are a promising candidate for highly sensitive biosensors owing to the excellent electronic and mechanical properties of SWNTs. Therefore, chemical sensors and biosensors based on CNTFETs have been extensively studied and investigated in academia and industry over the years. In this review, we will cover recent advances in the detection of biological species in various manners using CNTFETs. This article highlights the high-performance characteristics of CNTFETs, the detection of deoxyribonucleic acid (DNA) hybridization and proteins using CNTFET sensors and an effective measurement system for the highly sensitive detection of analytes, mainly focusing on works that were carried out in our laboratory.

\section{Introduction}

In recent years, the label-free electrochemical monitoring of biological events has been attracting much interest in various fields such as genomics, clinical diagnosis and practical pharmacy. In particular, there is an extensive need for compact, simple and cost-effective sensors that allow real-time measurement for home medical care. Therefore, there is still a great demand for highly sensitive and label-free biosensors based on nanomaterials.

${ }^{*}$ Corresponding author: e-mail: yasuki11@sanken.osaka-u.ac.jp 
Carbon nanotubes (CNTs) are one of the most attractive materials because of their unique molecular structure and well-defined nanoscale dimensions such as their extremely small diameter and unique carrier transportation. ${ }^{(1,2)}$ CNTs possess quasione-dimensional conductor or semiconductor characteristics with high current densities and high carrier mobilities. ${ }^{(3)}$ Therefore, CNTs are promising materials for advanced nanoelectronic devices, quantum-effect-based devices and microelectrodes. ${ }^{(4-11)}$ In particular, CNT field-effect transistors (CNTFETs) with single-wall carbon nanotube (SWNT) conducting channels are promising candidates for highly sensitive and labelfree biosensors and chemical sensors owing to their unique geometries with high surfaceto-volume ratio and high aspect ratio. In the case of SWNTs, every atom is on the surface and is exposed to the environment. Hence, even small changes in the charge environment can cause marked changes in their electrical properties. In addition to their diameters, which are comparable to the size of single molecules (e.g., $1 \mathrm{~nm}$ DNA in size), SWNTs are several micrometers long, thereby providing a convenient interface for micrometer-scale devices. All these superior characteristics make the CNTFET device configuration extremely sensitive to minute changes in the environment.

Sensor applications of CNTFETs have been investigated and discussed in several excellent papers. ${ }^{(12-16)}$ In this article, we discuss recent advances in the detection of biological and chemical species using CNTFETs, mainly focusing on studies carried out in our laboratory. In our studies, the electronic detections of biological and chemical species in a liquid environment have been explored. Our initial study demonstrated the effectiveness of CNTFET sensors and their excellent electrical properties for the precise and highly sensitive detection of biomolecules in solution. ${ }^{(17)}$ These properties of CNTFETs are suitable for the detection of various analytes. Therefore, two types of sensing using modified CNTFET sensors are described next. One is the detection of deoxyribonucleic acid (DNA) hybridization using CNTFETs with a peptide nucleic acid (PNA) probe-modified Au electrode, ${ }^{(18)}$ and the other is the label-free detection of immunoglobulin E (IgE) using aptamer-modified CNTFET biosensors. ${ }^{(19)}$ Finally, the effects of alternating current measurement on the noise suppression of CNTFET sensors were discussed. The techniques and properties of CNTFETs described here are expected to establish a foundation for the development of high-performance and highly sensitive sensors.

\section{High-Performance CNTFETs with Local Electrolyte Gates}

CNTFETs are well known to operate by the modulation of Schottky barriers between metals and SWNTs. ${ }^{(20)}$ If the sensing based on CNTFETs were primarily performed by Schottky barrier modulation in the SWNT-metal contact region, most of the channel region would not be used as an effective sensing area. To use the channel region of CNTFETs for biological sensing, the channel conductance modulation effect was reported. In this section, we describe CNTFETs with local electrolyte gates, in which switching occurs by the modulation of SWNT channel conductance. ${ }^{(17)}$ We discuss the advantages and excellent properties of local-electrolyte-gated CNTFETs. The electrical properties of the local-electrolyte-gated CNTFETs in a solution were compared with those of back-gated CNTFETs in air. 
After the devices were covered with a 300-nm-thick waterproof resist (ZEP520A), a 300-nm-wide groove was formed at the center of the SWNT channel by electronbeam lithography, as shown in Fig. 1(a). This groove leads to the formation of a local electrolyte gate in the solution, as mentioned above. The experiment was carried out using CNTFETs incubated in $10 \mathrm{mM}$ phosphate buffer solution (PBS). A saturated calomel reference electrode (BAS Inc.) was used as the top-gate electrode to minimize the effects of the environment. ${ }^{(21)}$ The electrical properties of the local-electrolyte-gated CNTFETs were measured with the top gate in PBS, while those of back-gated devices were all measured in air.

The gate transfer characteristics of the local-electrolyte-gated and back-gated CNTFETs were measured using the same SWNT devices, as shown in Fig. 1(b). The results revealed that the device exhibited p-type FET characteristics with the top gate in PBS as well as with the back gate in air. The local-electrolyte-gated CNTFET exhibited a sharp subthreshold characteristic compared with the back-gated CNTFET. Schottky barrier modulation via the resist caused by the electrolyte top gate was considered to be well-suppressed. Therefore, the good subthreshold characteristics of the localelectrolyte-gated devices shown in Fig. 1(b) were due to the channel-conductancemodulation effect. The subthreshold slopes of the back-gated and local-electrolytegated CNTFETs were estimated to be 190 and $60 \mathrm{mV} / \mathrm{dec}$, respectively. The slope of 60 $\mathrm{mV} / \mathrm{dec}$ was almost equal to the theoretical minimum. Therefore, the subthreshold slope was significantly improved in the local-electrolyte-gated device. Furthermore, in another fabricated CNTFET device, a high transconductance of $0.7 \mathrm{mS}$ at a drain voltage of $0.1 \mathrm{~V}$ was obtained using local-electrolyte-gated CNTFETs. This value was seven times higher than that obtained using back-gated CNTFETs. This result was due to the large gate capacitance of local-electrolyte-gated CNTFETs. Consequently, the high performance of the local-electrolyte-gated CNTFETs in the solution results from the modulation of the SWNT channel conduction and the larger gate capacitance. Local-electrolyte-gated CNTFETs with excellent electrical properties are suitable for biomolecule sensing.

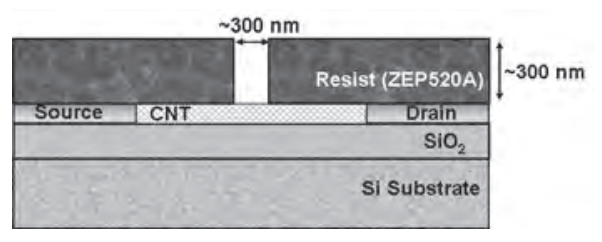

(a)

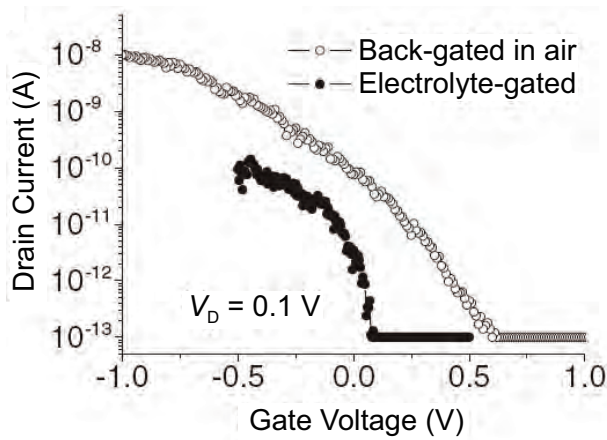

(b)

Fig. 1. (a) Schematic diagram of local-electrolyte-gated CNTFETs. (b) Gate transfer characteristics of local-electrolyte-gated CNTFET. 


\section{Biomolecule Detection Using CNTFETs}

In this section, we discuss two types of sensing using modified high-performance CNTFET sensors. One is the detection of DNA hybridization using CNTFETs with PNA probe-modified Au electrode, and the other is the label-free detection of IgE using aptamer-modified CNTFET biosensors.

\subsection{Sensing of DNA hybridization using CNTFETs}

Optical detection methods have commonly been used for detecting DNA hybridization, which are highly sensitive and specific methods. ${ }^{(22,23)}$ However, they require professional knowledge and techniques, and are very difficult to miniaturize. Therefore, alternative methods have been required, which are suitable for use in home medical diagnostics. In this section, we review the detection of DNA hybridization using CNTFETs. ${ }^{(18)}$ CNTFETs can be employed for the label-free, direct real-time electrical detection of biomolecule binding.

5'-end-amino-modified PNA oligonucleotide probes were covalently immobilized on the Au surfaces of the back gate of CNTFETs. PNA is a synthetic analog of DNA, in which both the phosphate and deoxyribose of the DNA backbone are replaced with a polypeptide. The advantages of using PNA instead of DNA in sensing systems are the presence of a neutral polypeptide backbone of PNA, which leads to improved PNA/DNA base-pairing free of ionic strength influence, and resistance to degradation by proteases and nucleases. ${ }^{(24)}$ CNTFET sensors with a microflow chip for the introduction of DNA samples were developed, as shown in Fig. 2(a). The microflow chip was fabricated using a poly (dimethylsiloxane) (PDMS) prepolymer. The CNTFET nanosensor array was placed onto the PDMS chip, and the PNA-probe-modified Au side of the CNTFET was oriented to face the open chamber containing DNA solutions. Electrical measurement was carried out using a semiconductor parameter analyzer connected to a $\mathrm{Ag} / \mathrm{AgCl}$ reference electrode immersed in $50 \mathrm{mM}$ PBS (pH 7.4).

The electrical characteristics of CNTFETs were monitored in real-time. Figure 2(b) shows the time dependence of the drain current of CNTFETs after the introduction of the target full-complementary DNA at $6.8 \mathrm{fM}$. A marked conductance increase was observed at the initial stage ( $\sim 20 \mathrm{~min})$. After a longer time scale, a marked and constant increase in conductance was observed for over $3 \mathrm{~h}$, reaching a level at which it remained stable. The increase in conductance for the p-type CNTFET device was consistent with the increase in negative surface charge density associated with the binding of negatively charged oligonucleotides at surfaces. ${ }^{(25)}$ The increase in conductance over a long period was attributed to the effects of the slow diffusion and binding constant of oligonucleotides at $\mathrm{fM}$ concentrations. Compared with other DNA hybridization biosensors, the label-free real-time detection system using CNTFETs has the advantages of eliminating tedious labeling steps and simplifying the analytical signal readout. 


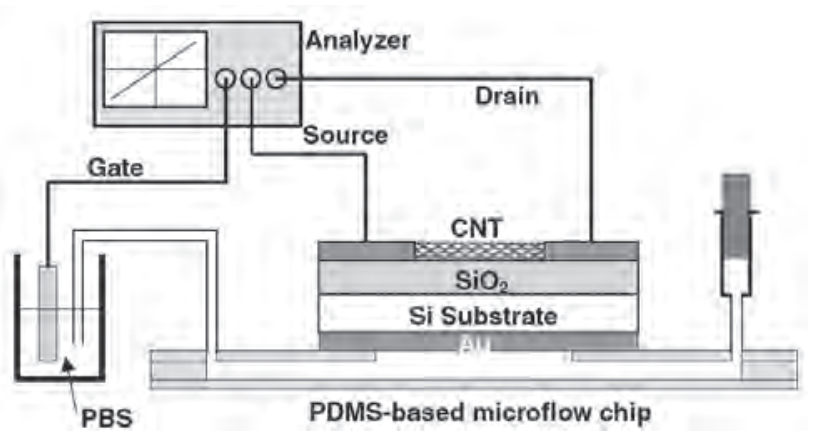

(a)

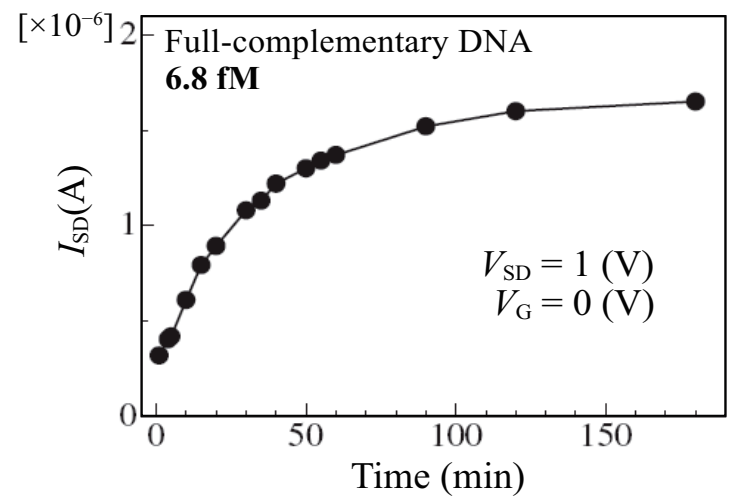

(b)

Fig. 2. (a) Schematic diagram of CNTFETs with microflow chip and electrical detection system. (b) Time dependence of drain current of CNTFET after the introduction of full-complementary target DNA at $6.8 \mathrm{fM}$.

\subsection{Label-free detection of protein using aptamer-modified CNTFETs}

In this section, we describe label-free protein biosensors based on aptamer-modified CNTFETs for IgE detection. ${ }^{(19)}$ After the covalent immobilization of 5'-amino-modified 45 mer aptamers on CNT channels, the electrical properties of the CNTFETs were monitored in real-time. The performance of this sensor was compared with that of CNTFET biosensors using a monoclonal antibody against $\operatorname{IgE}(\operatorname{IgE}-\mathrm{mAb})$.

Aptamers are synthetic oligonucleotides that can be generated to recognize amino acids, drugs and proteins with high specificity.(26,27) The aptamer used in our experiments is self-complementary with $12 \mathrm{bp}$. Therefore, it is significantly smaller than IgE- 
$\mathrm{mAb}$. Under similar ionic strength conditions, immunorecognition may have occurred outside the electrical double layer in solution, since the IgE-mAbs are much larger than the Debye length, as shown in Fig. 3(a). Thus, the charges of the bound protein may be screened by the double layer, and their effect on the equilibrium carrier distribution would then be negligible. On the other hand, aptamers enable sensitive detection, partly due to their small size. In terms of FET technology, aptamers are preferable because they are smaller than the Debye length, as shown in Fig. 3(b). As a result, binding between aptamers and target proteins can occur within the electrical double layer in buffer solution, and therefore, changes in the charge distribution within the proximity of the CNT can easily be detected.

The electrical properties of the CNTFETs were measured in real-time at room temperature using a semiconductor parameter analyzer. Figure 3(c) shows the time

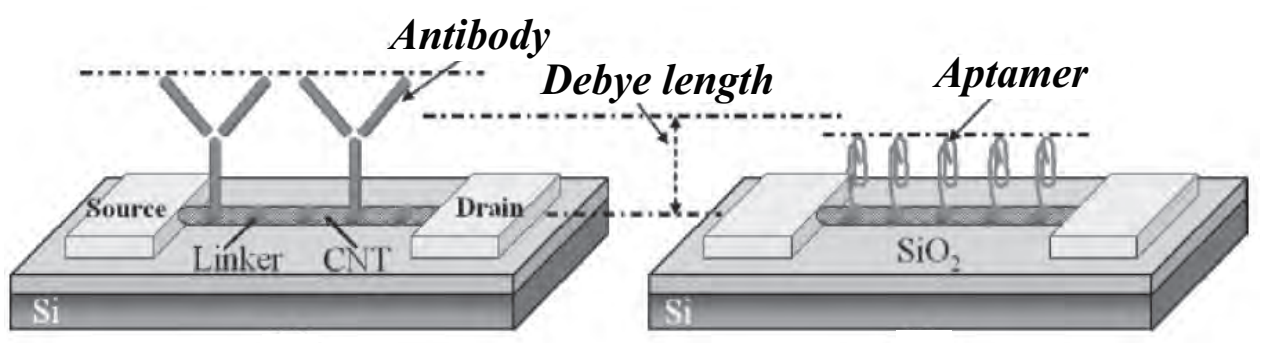

(a)

(b)

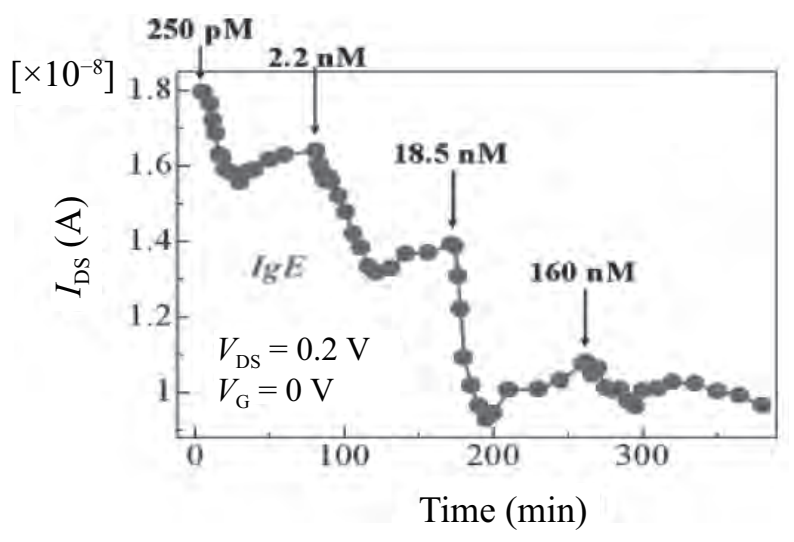

(c)

Fig. 3. (a) Schematic representation of antibody-modified CNTFETs. (b) Schematic representation of aptamer-modified CNTFETs. (c) Time dependence of drain current of CNTFET after the introduction of target $\mathrm{IgE}$ at various concentrations. Arrows indicate the points of $\operatorname{IgE}$ injections. 
dependence of the drain current of CNTFETs after the introduction of target $\operatorname{IgE}$ at various concentrations of $250 \mathrm{pM}, 2.2 \mathrm{nM}, 18.5 \mathrm{nM}$ and $160 \mathrm{nM}$ onto the aptamermodified CNTFET. Adding the target IgE caused a sharp decrease in drain current and then gradual saturation at lower values. This decrease was attributed to the screening effect of IgE molecules on the negative charge of the aptamers. The detection limit for IgE was determined as $250 \mathrm{pM}$ using the aptamer-modified CNTFET. However, after the addition of $160 \mathrm{nM}$ IgE into the chamber, a small change in conductance could be observed. Almost all the IgE aptamers were considered to be already bound with IgE molecules after the addition of $18.5 \mathrm{nM}$ IgE. Thus, the linear dynamic range was determined to be from $250 \mathrm{pM}$ to $20 \mathrm{nM}$. We suggest that the dynamic range of our biosensor makes it applicable for IgE detection. An aptamer-based CNTFET device is a promising candidate for the development of an integrated, high-throughput, multiplexed real-time biosensor.

\section{Noise Suppression in CNTFETs by Alternating Current Measurement}

CNTFETs often exhibit very large current fluctuations under a low-bias regime owing to the small diameter and large surface area of SWNT channels. ${ }^{(28-30)}$ They are also very sensitive to environmental factors and variations in device configuration, such as the charge of molecules ${ }^{(31)}$ and the nature of both SWNT-metal contacts ${ }^{(32)}$ and the interface of $\mathrm{SiO}_{2}$-SWNT. ${ }^{(33)}$ Moreover, the current fluctuations in the CNTFETs exhibit clear $1 / \mathrm{f}$ noise characteristics, which are considered to be caused by fluctuations in carrier mobility and carrier concentration. ${ }^{(34)}$ These factors are serious problems for CNTFET biosensors, because these sensors are generally used in the liquid phase in which very low bias is needed to avoid leakage current and redox reactions. To obtain highly sensitive sensors with satisfactory signal-to-noise ratios, the suppression of CNTFET noise is crucial. Here, we describe marked improvement in the sensitivity (i.e., an increased signal-to-noise ratio) of CNTFET sensors. ${ }^{(35)}$ To achieve this, alternating current (AC) measurement with a lock-in amplifier, which suppresses current fluctuations in the CNTFETs without decreasing signal level, was adopted. We report the noise suppression effect of the CNTFETs in buffer solutions determined by AC measurement.

Figure 4(a) shows the schematic cross section of a fabricated CNTFET and the experimental setup for AC measurement. AC measurement was carried out using a digital lock-in amplifier (NF; LI5640). The internal oscillator of the lock-in amplifier was used as an AC source, which was applied between the source and the drain. The modulation frequency in this work was set between 40 and $100 \mathrm{~Hz}$ with a time constant of $10 \mathrm{~ms}$ so that the fluctuations in drain current were minimized. A DC voltage was applied to the top- and back-gate electrodes using an Agilent $4156 \mathrm{C}$ precision semiconductor parameter analyzer. The time dependence of drain current was measured using an oscilloscope. For comparison, conventional DC measurement was also carried out using an Agilent 4156C analyzer.

The AC contribution to noise characteristics for CNTFET $\mathrm{pH}$ sensors was described. Figure 4(b) shows the time dependence of the drain current of the CNTFET pH sensor measured using both DC and AC. For AC measurement using the lock-in amplifier, the 


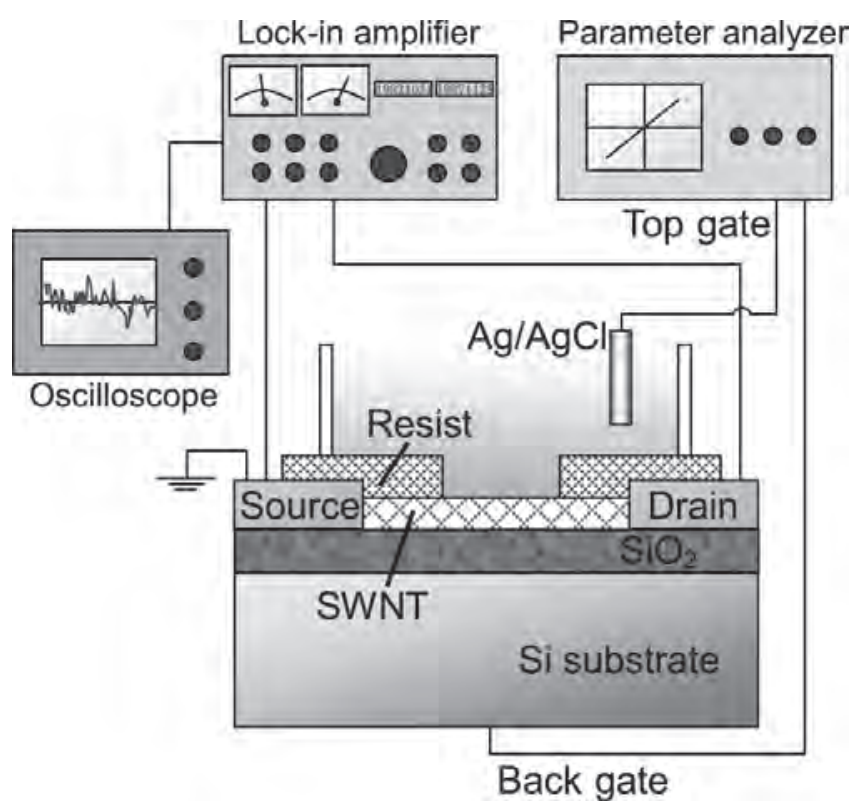

(a)

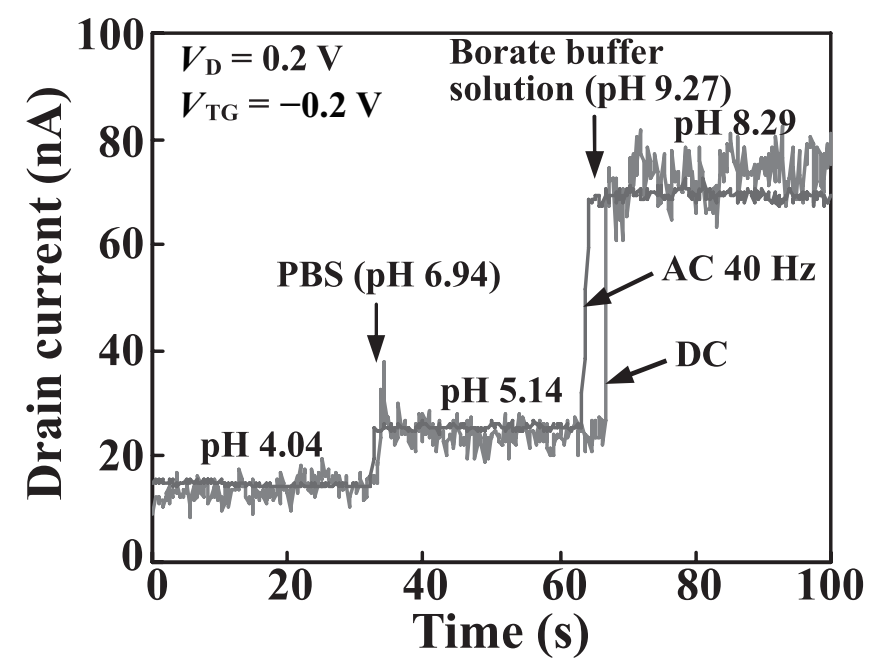

(b)

Fig. 4. (a) Schematic structure of CNTFETs and the experimental setup for AC measurement with a lock-in amplifier. (b) Time dependence of drain current. Arrows indicate the introduction of buffer solutions. The $\mathrm{pH}$ of solutions on CNTFET was changed from 4.04 to 8.29 through 5.14. 
effective drain voltage was $0.2 \mathrm{~V}$ with a frequency of $40 \mathrm{~Hz}$, and the top-gate voltage was $-0.2 \mathrm{~V}$. For DC measurement, the drain and top-gate voltages had the same values as those for $\mathrm{AC}$ measurement. During the monitoring of drain current fluctuations, the $\mathrm{pH}$ of the buffer solution was changed from 4.04 to 8.29 through 5.14 by adding buffer solutions with different $\mathrm{pH}$ values. By comparing the results obtained by DC measurement with those obtained by AC measurement, the noise level of the drain current measured by the latter method can be observed to be effectively and markedly reduced, although the signal level measured using $\mathrm{AC}$ is almost the same as that measured using DC. The noise level of the CNTFET pH sensor obtained using AC measurement was reduced to $1 / 6$ that obtained by DC measurement. Taking the signal level into account, the signal-to-noise ratio determined by $\mathrm{AC}$ measurement was increased six times as high as that determined by DC measurement. Therefore, AC measurement is effective and useful for application in CNTFET $\mathrm{pH}$ sensors. Furthermore, the detection of biomolecules using AC measurement was demonstrated. Bovine serum albumin (BSA; Sigma-Aldrich) with an arbitrary concentration dissolved in phthalate buffer solution ( $\mathrm{pH} 4$ ) was used as a target for sensing. The detection of a small amount of BSA (250 $\mathrm{pM}$ ) using $\mathrm{AC}$ measurement was successfully performed, which was difficult using DC measurement. Consequently, AC measurement is a promising technique for future highly sensitive sensor applications based on CNTFETs.

\section{Conclusions}

Recent advances in the development of biological and chemical sensors based on CNTFETs have been discussed, mainly focusing on works carried out in our laboratory. This area requires further development in the quantitative detection of a wide range of analytes and improvement in the fabrication of CNTFET devices. However, highly sensitive, label-free and noise-free detection of biological and chemical species was demonstrated. In the first study, local-electrolyte-gated CNTFETs, in which the channel conductance modulation effect is dominant in the switching, were fabricated, and the sensing utility of devices was investigated. BSA molecules were successfully detected by channel conductance modulation in local-electrolyte-gated CNTFETs. Localelectrolyte-gated CNTFETs exhibited high performance in electrical properties and sensing operation. Second, ultrasensitive real-time DNA hybridization detection was performed using CNTFETs functionalized with PNA probes on Au back-gate surfaces of the devices. The label-free detection of IgE using aptamer-modified CNTFET biosensors was described. Aptamers exhibited better performance for $\operatorname{IgE}$ detection than the monoclonal antibodies under similar conditions. Finally, a marked improvement in the sensitivity (signal-to-noise ratio) of CNTFET sensors was demonstrated. A decrease in the noise level of $\mathrm{pH}$ sensors without a decrease in signal level was demonstrated. Moreover, the detection of a small amount of BSA (250 pM) using AC measurement, which was difficult using DC measurement, was successfully performed. Such CNTFET devices are promising for future highly sensitive sensors. We consider that the sensitive multiplexed detection of numerous clinically important biomolecules will become possible in rapid and high-throughput format CNTFET sensors. 


\section{Acknowledgements}

The authors acknowledge the generous support from the Core Research for Evolutional Science and Technology (CREST), the Japan Science and Technology Corporation (JST), the New Energy and Industrial Technology Development Organization (NEDO), "Special Coordination Funds for Promoting Science and Technology: Yuragi Project" of the Ministry of Education, Culture, Sports, Science and Technology, Scientific Research from the Japan Society for the Promotion of Science, and Special Education, and Research Expenses (Post-Silicon Materials and Devices Research Alliance) from the Ministry of Education, Culture, Sports, Science and Technology of Japan.

\section{References}

1 S. Iijima: Nature 354 (1991) 56.

2 M. Dresselhaus, G. Dresselhaus and P. Avouris: Carbon Nanotubes: Synthesis, Structure, Properties, and Applications (Springer, Berlin, 2001).

3 Z. Yao, C. L. Kane and C. Dekker: Phys. Rev. Lett. 84 (2000) 2941.

4 S. J. Tans, A. R. M. Verschueren and C. Dekker: Nature 393 (1998) 49.

5 R. Martel, T. Schmidt, H. R. Shea, T. Hertel and P. Avouris: Appl. Phys. Lett. 73 (1998) 2447.

6 D. Kaminishi, H. Ozaki, Y. Ohno, K. Maehashi, K. Inoue, K. Matsumoto, Y. Seri, A. Masuda and H. Matsumura: Appl. Phys. Lett. 86 (2005) 113115.

7 M. Bockrath, D. H. Cobden, P. L. McEuen, N. G. Chopra, A. Zettl, A. Thess and R. E. Smalley: Science 275 (1997) 1922.

8 K. Maehashi, H. Ozaki, Y. Ohno, K. Inoue, K. Matsumoto, S. Seki and S Tagawa: Appl. Phys. Lett. 90 (2007) 023103.

9 S. J. Tans, M. H. Devoret, H. Dai, A. Thess, R. E. Smalley, L. J. Geerligs and C. Dekker: Nature 386 (1997) 474.

10 J. Okuno, K. Maehashi, K. Kerman, K. Matsumoto, Y. Takamura and E. Tamiya: Biosens. Bioelectron. 22 (2007) 2377.

11 Y. Tsujita, K. Maehashi, K. Matsumoto, M. Chikae, S. Torai, Y. Takamura and E. Tamiya: Jpn. J. Appl. Phys. 47 (2008) 2064.

12 A. Star, J. C. P. Gabriel, K. Bradley and G. Gruner: Nano Lett. 3 (2003) 459.

13 K. Besteman, J. Lee, F. Wiertz, H. Heering and C. Dekker: Nano Lett. 3 (2003) 727.

14 C. Li, M. Curreli, H. Lin, B Lei, F. N. Ishikawa, R. Datar, R. J. Cote, M. E. Thompson and C. Zhou: J. Am. Chem. Soc. 127 (2005) 12484.

15 E. L. Gui, L. J. Li, P. S. Lee, A. Lohani, S. G. Mhaisalkar, Q. Cao, S. J. Kang, J. A. Rogers, N. C. Tansil and Z. Gao: Appl. Phys. Lett. 89 (2006) 232104.

16 H. R. Byon and H. C. Choi: J. Am. Chem. Soc. 128 (2006) 2188.

17 T. Katsura, Y. Yamamoto, K. Maehashi, Y. Ohno and K. Matsumoto: Jpn. J. Appl. Phys. 47 (2008) 2060 .

18 K. Maehashi, K. Matsumoto, K. Kerman, Y. Takamura and E. Tamiya: Jpn. J. Appl. Phys. 43 (2004) L1558.

19 K. Maehashi, T. Katsura, K. Karman, K. Matsumoto and E. Tamiya: Anal. Chem. 79 (2007) 782.

20 S. Heinze, J. Tersoff, R. Martel, V. Derycke, J. Appenzeller and Ph. Avouris: Phys. Rev. Lett. 89 (2002) 106801. 
21 E. D. Minot, A. M. Janssens and I. Heller: Appl. Phys. Lett. 91 (2007) 093507.

22 E. Palecek: Trends Biotechnol. 22 (2004) 55.

23 J. H. Watterson, P. A. E. Piunno and U. J. Krull: Anal. Chim. Acta 457 (2002) 29.

24 M. Egholm, O. Buchardt, L. Christensen, C. Behrens, S. M. Freier, D. A. Driver, R. H. Berg, S. K. Kim, B. Norden and P. E. Nielsen: Nature 365 (1993) 566.

25 J.-I. Hahm and C. M. Lieber: Nano Lett. 4 (2004) 51.

26 C. Tuerk and L. Gold: Science 249 (1990) 505.

27 P. M. Robertson and F. G. Joyce: Nature 344 (1990) 467.

28 P. Collins, M. Fuhrer and A. Zettl: Appl. Phys. Lett. 76 (2000) 894.

29 E. Snow, J. Novak, M. Lay and F. Perkins: Appl. Phys. Lett. 85 (2004) 4172.

30 F. Liu, L. Wang, D. Zhang and C. Zhou: Appl. Phys. Lett. 89 (2006) 063116.

31 J. Mannik, I. Heller, A. M. Janssens, S. G. Lemay and C. Dekker: Nano Lett. 8 (2008) 685.

32 J. Appenzeller, Y. M. Lin, J. Knoch, Z. Chen and P. Avouris: IEEE Trans. Nanotechnol. 6 (2007) 368.

33 M. Briman, K. Bradley and G. Gruner: J. Appl. Phys. 100 (2006) 013505.

34 Y. M. Lin, J. Appenzeller, J. Knoch, Z. Chen and P. Avouris: Nano Lett. 6 (2006) 930.

35 Y. Yamamoto, Y. Ohno, K. Maehashi and K. Matsumoto: Jpn. J. Appl. Phys. 48 (2009) 06FJ01. 